\title{
El uso de tocilizumab en pacientes con COVID-19. Consideraciones metodológicas y éticas
}

\section{The use of tocilizumab in patients with COVID-19. Methodological and ethical considerations}

\author{
Octavio Martínez-Michel, ${ }^{*}$ Ireri Isadora Romero-Thirión ${ }^{\ddagger}$
}

\author{
*Universidad del Claustro de Sor Juana, ${ }^{\ddagger}$ Instituto Nacional de Enfermedades Respiratorias \\ Ismael Cosío Villegas, Ciudad de México.
}

Palabras clave: COVID-19, tocilizumab, dilemas éticos, pandemia. Keywords: COVID-19, tocilizumab, ethical dilemmas, pandemic.

«... cuando la teoría sirve de poco para la práctica, esto no se debe achacar a la teoría, sino al hecho de que no había bastante teoría...» Immanuel Kant

Desde que la COVID-19 fue descubierta el debate público alrededor de las vacunas y los posibles tratamientos para curarla han sido muy intensos tanto a nivel nacional como internacional. A medida que los contagios fueron avanzando y el control de la pandemia complicándose, la información disponible al respecto también se ha multiplicado. La pandemia es fundamentalmente una cuestión de salud pública, pero que ha trastocado la economía mundial, así como los equilibrios sociales (ya de por sí endebles). Ante ello, la urgencia por encontrar una vacuna o una cura se ha vuelto cada vez más apremiante, lo que ha generado una explosión en los estudios clínicos.

Entre los muchos fármacos que han estado bajo el escrutinio está el tocilizumab, medicamento con posible efecto benéfico para el tratamiento del llamado síndrome de tormenta de citocinas. A nivel nacional el debate se volvió

Correspondencia:

Dra. Ireri Isadora Romero-Thirión

Instituto Nacional de Enfermedades Respiratorias

Ismael Cosío Villegas, Ciudad de México.

Correo electrónico: draisadora thirion@hotmail.com

Recibido: 03-III-2021; aceptado: 04-III-2021.

Citar como: Martínez-Michel O, Romero-Thirión II. El uso de tocilizumab en pacientes con COVID-19. Consideraciones metodológicas y éticas. Neumol Cir Torax. 2021; 80 (1): 6-8. https://dx.doi.org/10.35366/99447 particularmente relevante después del comunicado de la Secretaría de Salud en el que se afirma que el tocilizumab no debe utilizarse como tratamiento alternativo para la COVID-19 y que su uso debería circunscribirse a ensayos clínicos controlados. En el debate se han escrito argumentos cuestionando la decisión de la Secretaría de Salud bajo el supuesto de que hay suficiente evidencia científica para autorizar un uso más amplio del citado fármaco.

Desde nuestra perspectiva no sólo carecemos de evidencia científica contundente que nos permita afirmar que el tocilizumab debería utilizarse en nuestro país de forma más amplia, sino que su uso fuera de un ensayo clínico nos lleva a complicaciones éticas en las que no hay una salida clara. En ese sentido, consideramos pertinente entrar a este debate aportando perspectivas metodológicas y éticas que puedan colocar los riesgos y beneficios del tocilizumab en una justa balanza.

\section{CONSIDERACIONES METODOLÓGICAS}

El síndrome de tormenta de citocinas se ha observado en un porcentaje de los pacientes con enfermedad por COVID-19 que oscila entre 10 y $20 \%$, el síndrome se describe como un estado sistémico de hiperinflamación potencialmente letal. Su identificación se basa en el deterioro respiratorio rápido y progresivo, fiebre elevada y persistente, niveles elevados de proteína $C$ reactiva y ferritina de forma desproporcionada. Es por ello que el tocilizumab ha sido propuesto como agente terapéutico por su probable respuesta benéfica en el manejo de la tormenta de citocinas., ${ }^{1,2}$

$\mathrm{Al}$ respecto, vale la pena anotar que la mayor parte de la evidencia publicada para el uso de tocilizumab en pacientes con infección por COVID-19 en los que se sospecha la presencia del síndrome consiste en lo siguiente: estudios retrospectivos, unos cuantos estudios prospectivos, 
otros ensayos clínicos abiertos y no controlados, y muy pocos, hasta el momento, ensayos clínicos aleatorizados y cegados. ${ }^{3}$

Identificar el grado y nivel de evidencia que tenemos hasta el momento nos ayuda a disminuir nuestros errores en la interpretación de los resultados. Por ejemplo, en Clinical Trials se encuentran registrados diversos estudios, algunos de ellos aún en reclutamiento, otros ya finalizados pero sin publicación de resultados y otros retirados. ${ }^{4}$ Todo ello es un indicador de la cautela que debemos tener aún con la administración del tocilizumab.

Ahora, si nos atenemos a estudios con mejor nivel de evidencia, tampoco encontramos resultados que nos permitan concluir grandes beneficios en el uso del tocilizumab. Por ejemplo, el de Stone JH et al., publicado en noviembre de 2020, concluye que el uso de tocilizumab en pacientes con COVID-19 que reportaban fiebre, infiltrados pulmonares o requerían oxígeno para mantener saturación por arriba de 92\% no reportó diferencias significativas comparadas con el tratamiento estándar en la disminución de mortalidad y días de ventilación mecánica. En el estudio se reportó disminución en el número de días de hospitalización, lo que supone evidencia significativa a favor del fármaco mencionado, aunque vale la pena anotar que esto último no se encontraba dentro de los objetivos primarios del estudio, por lo que también habría que tomar con cautela la evidencia. ${ }^{5}$

Después, en diciembre de 2020, se publicó un ensayo clínico controlado en pacientes que presentaban saturación aire ambiente por debajo de $94 \%$, pero que no requerían el uso de ventilación mecánica (VM). En él se encontró que el riesgo de requerir $\mathrm{VM}$ al día 28 era menor en el grupo de tocilizumab. A pesar de ello, no se reportó una diferencia significativa en la mortalidad a 60 días de seguimiento; se reportaron $11.6 \%$ de muertes en el grupo de tocilizumab y $11.8 \%$ en el grupo de placebo. ${ }^{6,7}$

Gracias a estos últimos estudios con buen nivel de evidencia la recomendación del uso de tocilizumab en pacientes con COVID-19 se plantea dentro de un ensayo clínico controlado, con un grado de recomendación moderado y nivel de evidencia I. ${ }^{8}$ Así, la evidencia disponible nos obliga como comunidad científica a ser prudentes a la hora de sugerir el uso del fármaco. Además, como se hace evidente con los últimos trabajos publicados, aunque son ensayos clínicos abiertos, se realiza la recomendación del uso de tocilizumab junto con dexametasona en pacientes con ciertas características, con un nivel de recomendación moderado y nivel de evidencia Ila. ${ }^{8,9,10}$

\section{CONSIDERACIONES DEONTOLÓGICAS}

Es cierto que quienes nos desempeñamos en la medicina clínica debemos evaluar si las intervenciones son útiles o no en nuestro paciente, la clínica opera en el caso concreto. Sin embargo, el sustento científico y práctico que apoya nuestras decisiones sobre el uso de algún tratamiento tiene que generar un beneficio objetivo para el paciente. Como hemos afirmado en los párrafos anteriores, la evidencia objetiva respecto al beneficio del uso del tocilizumab es limitada.

Por otra parte, es necesario cuestionarse si un escenario de desgaste profesional y emocional para el personal de salud y de pánico persistente para la población es un terreno fértil para la objetividad científica. La situación de crisis que vivimos nos conduce a callejones oscuros. Así, en un estado de ceguera y ansiedad, ofrecer una oportunidad de esperanza al paciente se nos presenta como el único camino con luz que encontramos. El problema es que esa luz puede ser sólo una apariencia que se desvanece apenas le aplicamos un método ético y científico riguroso.

Esto nos obliga a reflexionar sobre cuáles son nuestros alcances, qué es lo que podemos ofrecer al paciente para evitar su muerte y qué podemos darles a aquellos cuya muerte es inevitable. Como profesionales de la medicina tenemos la obligación de ofrecerle al paciente un tratamiento que tenga siempre mayor beneficio que perjuicio. Por ello, es imperioso reconocer la situación de vulnerabilidad social en la que nos encontramos y realizar un ejercicio continuo de autoanálisis para evitar que la urgencia nos lleve a sugerir tratamientos que aún no están suficientemente probados. No existe justificación ética para especular con los pacientes.

Ahora bien, en el debate público sobre el uso del tocilizumab se ha dicho que la protección y preservación de la vida representa el bien supremo de la práctica de la medicina. Esto es cierto, pero ello no puede llevarnos a concluir que la vida deba concebirse como un absoluto, pues el valor de la vida está relacionado también con la dignidad y con la posibilidad de poder vivir sanamente. Por ello, Vallejo Rubiola nos recuerda que la intervención médica es válida «si alivia objetivamente el dolor humano (físico o psíquico) y sus capacidades curativas superan los eventuales efectos adversos que toda terapéutica activa conlleva. Cualquier otra consideración que se aparte de estos criterios entra en el terreno de la ignorancia o la irresponsabilidad»..11

La responsabilidad de quien ejerce la medicina, tal y como nos recuerda Savater, reside en intentar prestar ayuda ante un problema que es científico, social y metafísico, ${ }^{12}$ es decir, que el problema al que se enfrentan las y los profesionales de la medicina no puede resolverse de forma adecuada si no se tienen investigaciones solventes, si se carece de entendimiento sobre el valor específico que las sociedades dan a la vida o si se ignora la complejidad de la propia definición de la vida.

También se ha citado en el debate público sobre el uso del tocilizumab a nuestra Constitución Política, arguyendo 
que ésta protege el derecho a la vida. Esto también es cierto, pero es importante considerar que tanto nuestra Constitución como los tratados internacionales de derechos humanos protegen el derecho a la salud y la dignidad humana. Esos tres horizontes se entrelazan para construir un concepto de vida que va más allá de su dimensión biológica, para entenderla como una dimensión cultural y moral.

Así, es importante recordar que, según el Plan Beveridge, desde los años 40 del siglo XX, la salud se ha transformado en objeto de preocupación de los Estados, es decir, el derecho de los seres humanos a mantener su cuerpo en buena salud se ha convertido en objeto de acción del estado. Desde que inició ese proceso hemos aprendido que cuando se trata de salud nunca será suficiente el mero consenso estratégico, sino que es indispensable el consenso racional. También nos ha permitido recordar que a veces podemos olvidar que el objetivo básico es encontrar una solución éticamente correcta. ${ }^{13}$ De tal suerte que la vida humana no puede instrumentalizarse, ni supeditarse a éticas utilitaristas.

Son muchos los retos que la COVID-19 nos ha puesto en el camino. Para la medicina, uno especialmente relevante es la administración de fármacos que teóricamente podrían generar resultados alentadores en un contexto de tragedia e incertidumbre. Sin embargo, ese reto no puede superarse de forma adecuada si se pierde de vista que, frente a la duda del beneficio de los fármacos, tendríamos que ofrecer el mejor diseño de investigación para poder comprobar nuestras hipótesis y seguir nuestro imperativo ético primum non nocere (lo primero es no hacer daño).

Hannah Arendt en su famoso texto «La condición humana», nos recordaba que uno de los dilemas éticos más complejos tenía que ver con la imposibilidad de predecir los efectos de nuestras acciones. ${ }^{14}$ Según la filósofa alemana, una vez que realizamos una acción ésta sale de nosotros y su efecto es imposible de prever. Con ello, nos advertía del cuidado escrupuloso que debíamos tener con nuestras acciones en el terreno de la moral, esto es especialmente relevante para profesiones donde lo que está en juego es la vida, la libertad o la dignidad. En la práctica de la medicina, están en juego las tres, de tal suerte que cualquier tratamiento o cura que ofrezcamos debería cumplir un estándar ético mínimo en el que podamos prever que la vida, la dignidad o la libertad no están sujetas a los designios de la diosa Fortuna.

\section{REFERENCIAS}

1. Ragab D, Salah Eldin H, Taeimah M, Khattab R, Salem R. The COVID-19 cytokine storm; what we know so far. Front Immunol. 2020;11:1446. https://doi.org/10.3389/fimmu.2020.01446

2. Moore JB, June $\mathrm{CH}$. Cytokine release syndrome in severe COVID-19. Science. 2020;368(6490):473-474. https://doi.org/10.1126/science. abb8925

3. Cortegiani A, Ippolito M, Greco M, Granone V, Protti A, Gregoretti C, et al. Rationale and evidence on the use of tocilizumab in COVID-19: a systematic review. Pulmonology. 2021;27(1):52-66. https://doi. org/10.1016/j.pulmoe.2020.07.003

4. Clinical Trial. Tocilizumab in COVID-19 pneumonia (TOCIVID-19). [Access date: 2021 January 6] Available from: https://www. clinicaltrials.gov/ct2/results?cond=covid-19\&term=TOCILIZUMAB\& cntry $=\&$ state $=\&$ city $=\&$ dist $=$

5. Stone JH, Frigault MJ, Serling-Boyd NJ, Fernandes AD, Harvey L, Foulkes AS, et al. Efficacy of tocilizumab in patients hospitalized with COVID-19. N Engl J Med. 2020;383(24):2333-2344. https://doi. org/10.1056/nejmoa2028836

6. Salama C, Han J, Yau L, Reiss WG, Kramer B, Neidhart JD, et al. Tocilizumab in nonventilated patients hospitalized with Covid-19 pneumonia. medRxiv [Internet]. 2020;(650):2020.10.21.20210203. Available from: https://doi.org/10.1101/2020.10.21.20210203

7. Huang E, Jordan SC. Tocilizumab for Covid-19-The ongoing search for effective therapies. N Engl J Med. 2020;383(24):2387-2388. https:// doi.org/10.1056/nejme2032071

8. NIH. The COVID-19 Treatment Guidelines Panel's Statement on the Use of Tocilizumab for the Treatment of COVID-19. [Access date: 2021 January 6] Available from: https://www.covid19treatmentguidelines. nih.gov/immune-based-therapy/immunomodulators/

9. REMAP-CAP Investigators, Gordon AC, Mouncey PR, Al-Beidh F, Rowan KM, Nichol AD, et al. Interleukin-6 Receptor Antagonists in Critically III Patients with Covid-19. N Engl J Med. 2021;1-12. doi: 10.1056/NEJMoa2100433.

10. RECOVERY Collaborative Group, Horby PW, Pessoa-Amorim G, Peto L, Brightling CE, Sarkar R, et al. Tocilizumab in patients admitted to hospital with COVID-19 (RECOVERY): preliminary results of a randomised, controlled, open-label, platform trial. medRxiv 2021.02.11.21249258; doi: https://doi.org/10.1101/2021.02.11.21249258.

11. Vallejo RJ. Mito y realidad de la terapéutica electroconvulsiva. Med Clin Barcelona. 1983;(81):345-346.

12. Savater F. "Cuidar al prójimo: compasión con pasión" ante el dolor reflexiones para afrontar la enfermedad y la muerte (Prólogo). Madrid: Temas de hoy; 2000. p. 13.

13. Couceiro VA. Comités de ética y eutanasia. Madrid: Noesis; 1996. p. 293-312.

14. Arent H. La condición humana. Barcelona: Paidós; 2005. p. 255-265.

Conflicto de intereses: Los autores declaran no tener conflicto de intereses. 